\title{
Correction to: Repurposing anaerobic digestate for economical biomanufacturing and water recovery
}

\author{
Santosh Kumar ${ }^{1} \cdot$ Roy Posmanik ${ }^{2} \cdot$ Sabrina Spatari ${ }^{3} \cdot$ Victor C. Ujor $^{1}$ \\ Published online: 17 February 2022 \\ ๑) Springer-Verlag GmbH Germany, part of Springer Nature 2022
}

\section{Correction to: Applied Microbiology and Biotechnology https://doi.org/10.1007/s00253-022-11804-6}

The published version of the article contained some errors.

In the Concluding remarks section, the text were inadvertently omitted in the PDF during correcting process.

The Concluding remarks text should read as below:

Due to depleting global mineral reserves and increasing pressure on water resources, there is an urgent need to valorize nutrient-laden and water-replete wastes such ADE beyond land application. As the impacts of climate change continue to worsen, adept measures that recover valuable water from ADE demand increased attention. Deployment of ADE as a resource in biomanufacturing of high-value chemicals has substantial promise to offset the costs associated with this approach. Thus, harnessing ADE-borne nutrients for economical production of bio-chemicals while recovering valuable water and, possibly, increasingly important phosphate for reuse deserves significant research attention. Increase in the incidence of desertification globally and water scarcity should be a trigger that highlights the urgency to develop novel approaches and technologies for expanded recovery of water from currently untreated effluents in addition to

The original article can be found online at https://doi.org/10.1007/ s00253-022-11804-6.

Victor C. Ujor

ujor@wisc.edu

1 Fermentation Science Group, Department of Food Science, Babcock Hall, University of Wisconsin-Madison, 1605 Linden Drive, Madison, WI 53706, USA

2 Agricultural Research Organization, Volcani Institute Newe Ya' ar Research Center, Rishon LeZion, Israel

3 Faculty of Civil and Environmental Engineering, Technion, Israel Institute of Technology, Haifa, Israel specialized wastewater treatment plants that will recover potable water from sewage. The mineral and buffer requirements for glutamine, glutamate, fumarate, lactate, and succinate production represent early research targets for likely deployment of ADE as a nutrient source in biomanufacturing. More importantly, the expanding field of synthetic biology holds great promise for rewiring nutrient transport machineries in select microorganisms for efficient nutrient removal from $\mathrm{ADE}$ (to recover valuable water), while producing high-value chemicals and accumulating recoverable phosphate. Indeed, if ADE is to transition from the laboratory to industry, certain key hurdles still need to be circumvented. First, there is need to design systems that eliminate and/or contain the foul odor associated with ADE. Second, a measure that allows for economical sterilization of ADE without precipitating the valuable nutrients essential to the fermentation process needs to be developed. Third, given the wide variety of nutrients in ADE (that need to be removed), coupled with the need to engineer strains that can produce high amounts of target products during growth in ADE, engineering functional microbial consortia, such that different strains perform specific tasks during growth in ADE, is a likely pre-requisite for efficient and economical treatment of ADE that demands research attention. Lessons learned from successful ADE-aided biomanufacturing would most certainly inform and engender similar efforts for addressing other nutrient-replete, water-rich effluents such as landfill leachate, tannery, and largescale fish processing effluents as potential feedstocks in fermentative biomanufacturing.

This is being corrected in this publication.

Publisher's note Springer Nature remains neutral with regard to jurisdictional claims in published maps and institutional affiliations. 